\title{
Enhanced Traffic Simulation for Improved Realism in Driving Simulators
}

Bihao Wang

Marwa Mahmoud

Department of Computer

Science and Technology

University of Cambridge

Cambridge, CB30FD, UK

name.surname@cl.cam.ac.uk

Javier Echevarría Cuesta

Stanford University

California, USA

javierec@stanford.edu
Hannah Close

University of Cambridge

Cambridge, CB30FD, UK

hlc64@cam.ac.uk

Quentin Stafford-Fraser Peter Robinson

Department of Computer

Science and Technology

University of Cambridge

Cambridge, CB30FD, UK

name.surname@cl.cam.ac.uk

Permission to make digital or hard copies of all or part of this work for personal or classroom use is granted without fee provided that copies are not made or distributed for profit or commercial advantage and that copies bear this notice and the full citation on the first page. Copyrights for components of this work owned by others than the author(s) must be honored. Abstracting with credit is permitted. To copy otherwise, or republish, to post on servers or to redistribute to lists, requires prior specific permission and/or a fee. Request permissions from permissions@acm.org. Copyright held by the owner/author(s). Publication rights licensed to ACM. AutomotiveUI '18 Adjunct, September 23-25, 2018, Toronto, ON, Canada ACM 978-1-4503-5947-4/18/09.

\begin{abstract}
The ability to monitor and detect potentially dangerous behaviour in surrounding traffic is vital for the development of intelligent vehicles. However, data collection for these kinds of scenarios is difficult in real-life, and a driving simulator therefore becomes an important substitute. In this paper we present an approach to enhance driving simulators. We experiment on an open source development platform, which is used to test real-life use cases within a simulated vehicle environment. We propose replacing pre-programmed traffic dynamics with real driving data recorded from human drivers in the same environment. This enhances the engagement of the host driver in the more realistically simulated traffic scenario. Signal lights and indicator sounds are also integrated to enrich the driver's sensation. Our preliminary quantitative and qualitative evaluation shows that our enhanced traffic simulation results in an improvement to the driver's perception of the realism of the driving simulator.
\end{abstract}

\section{Author Keywords \\ Driver experience; driving simulator; traffic realism.}

\section{CCS Concepts}

-Human-centered computing $\rightarrow$ Human computer interaction $(\mathrm{HCl}) ; \mathrm{HCl}$ design and evaluation methods; Scenariobased design; •Applied computing $\rightarrow$ Transportation; 


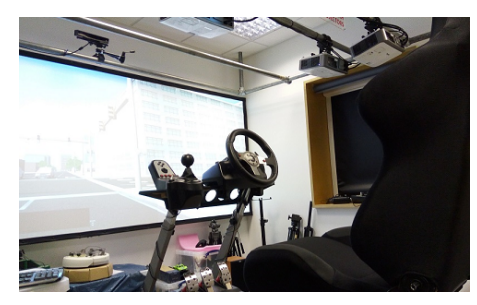

Figure 1: Driving simulator: equipment set-up

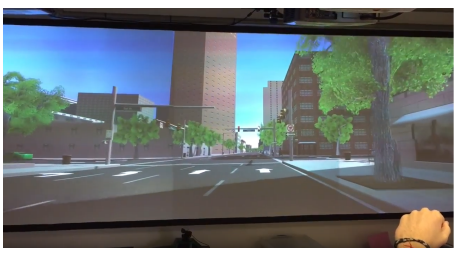

Figure 2: Driving simulator: experiment scenario

\section{Introduction}

Safety and reliability are perhaps the most-discussed topics in intelligent transportation research. Recognising the characteristics of other vehicles' and drivers' behaviour will greatly improve safety on the road. However, collecting data from risky situations in real traffic can be life-threatening, and gathering realistic samples of dangerous driving characteristics in surrounding vehicles can be prohibitively timeconsuming. A simulator may therefore be a viable choice $[8,2]$. Despite the reasonably high-quality synthetic scenarios [3] provided by modern systems, they usually don't contain as many difficult traffic situations as in the real world. For example, in current simulators, non-player vehicles in traffic always obey the traffic regulations. Can we make simulators as representative of real world dynamics as possible?

We experiment using the GENIVI vehicle simulator [1], which is an Open Source software project that is extensible for various applications and research purposes. In an attempt to make it more closely represent a real driving scenario, we replace the pre-programmed surrounding traffic with vehicles using driving/motion data of human drivers that are previously recorded. This aims to bring a sense of unpredictable dynamics, as in real traffic. We also add functions to provide indicator sounds and the visualisation of braking and signal lights to engage the driver's attention and senses. Most importantly, synchronisation and collision avoidance functions are developed for the traffic simulation using real driving data.

In this paper, we describe our proposed enhancements and present a qualitative and quantitative evaluation of their effects on the driving experience. We show that introducing real driving data in traffic simulations can result in significant improvement in the driver's perception of the realism of the simulator.

\section{Related work}

One of the most recent simulators, CARLA [3], is developed for generating the synthetic data of multi-sensors, so the data can be used to train autonomous driving models. Some other work focuses on the effect of enhancing the simulator to get as close as possible to a real-world driving experience. Wade et al. [5] compared two scenarios of driving in the real world with driving in the same environment in a simulator. They used two post-experiment questionnaires that evaluated physical as well as mental comfort and realism. Using similar questionnaires, Michael et al. [4] evaluated the ease of use and the degree of immersion.

Most of this previous work focused on improving the visual environment and roads where the driving takes place [7]. In our work, we examine instead the effect of changing the functionality and behaviour of the surrounding vehicles. An experimental design is deployed (questionnaires) to evaluate the effect of the proposed changes. We found that replaying previous driving recordings in the scenario makes participants feel as if there were real humans driving around whose behavior was unpredictable and demanded the participant's attention.

\section{System development}

The purpose of the study is to build an interactively realistic simulator that can be used for collecting driving data from traffic scenarios that may be dangerous in the real world. We selected the GENIVI vehicle simulator because it is Open Source and allows easy implementation of various functions for research purposes. It includes a road map of part of San Francisco as its urban driving scene. Like most simulators, the traffic surrounding is predefined by simple dynamic models (e.g. cars with constant speed on designated routes). In order to improve the driver's experience, we replaced program-generated traffic with previouslyrecorded driving data from human drivers. 


\begin{tabular}{|l|}
\hline Experimental Setup \\
(hardware) \\
6 projectors are aligned to \\
produce a single high reso- \\
lution display of $4 \times 1.5 \mathrm{~m}[6]$. \\
The car seat equipped with \\
Logitech driving controls is set \\
at a distance of $2.5 \mathrm{~m}$ to the \\
screen. The simulator runs on \\
a Windows 10 PC with dual \\
2.4 GHz processors, three \\
NVIDIA Quadro K5000 GPUs, \\
and 16 GB RAM. \\
\hline
\end{tabular}

\section{Experimental Setup}

6 projectors are aligned to produce a single high resodisplay of $4 \times 1.5 \mathrm{~m}[6]$ at a distance of $2.5 \mathrm{~m}$ to the screen. The simulator runs on and 16GB RAM.
Driving data collection and replay

We modified GENIVI to record the host vehicle data (position, steering, speed, braking, indicators) from participants driving on the simulator. Then we added a function to play recordings back in the simulator and use each to control the behaviour of a simple vehicle model. By doing this, more unpredictable events are introduced in the traffic simulation, such as: lane changing, sudden braking, and red-lightrunning. These types of events would be more complex to program realistically and so are rarely seen in simulators, but can be easily implemented by replaying human driving data.

Indicator and signal lights

In order to engage better the driver's visual and auditory senses, we enhanced a standard vehicle model so it could render brake and signal lights (instead of using expensive commercial car models). We use the steering-columnmounted gear change paddles as turn indicators, and added a realistic clicking sound similar to that found in a typical car when they are in use. During the replay of a recording, we render the indicator and the brake lights as they were used in the original recording which, again, adds to the realism of the replay. For example, an earlier lost driver might use a wrong signal light in his recording, and this requires the judgment of current driver from his observation.

Collision avoidance and synchronisation

The system was designed to record multiple drivers' behavior and then incorporate their data in a later scenario to build up traffic. This could lead to a collision problem if the trajectory of two vehicles overlap, especially if the host vehicle is in a location which was empty at the time of a recording. Each new driver added could see earlier recorded vehicles, so they seldom collided with them; the problem is that these earlier vehicles could not see the current driver. So, firstly, we synchronised all the recordings from differ-

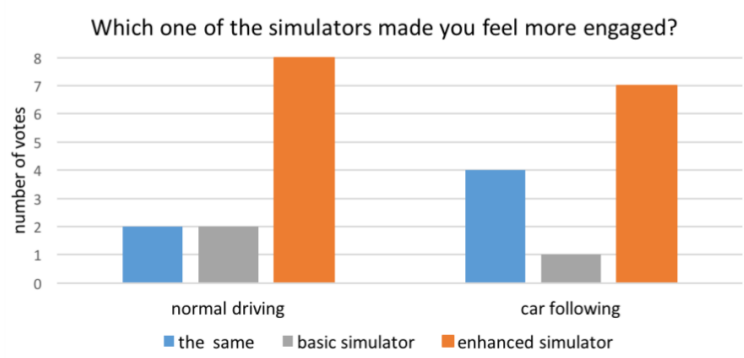

Figure 3: The Enhanced simulator made drivers feel more engaged during normal (free) driving.

ent drivers. We also synchronised the recordings with the global clock used, for example, for traffic light changes, so replayed vehicles would still stop when the lights turned red. Then, if a recorded vehicle is about to collide with the host vehicle - if approaching from behind with a higher speed its behavior is adjusted by slowing - and if necessary pausing it for several seconds, before resuming its original trajectory.

\section{Experimental evaluation}

As a preliminary evaluation, twelve volunteers were invited from different genders with variant driving experiences (see Fig. 4 and Fig. 5). We divided the participants into two groups of six people each. The first group starts with driving the non-improved 'Basic' simulator, then driving the 'Enhanced' simulator. The second group drive on the two simulators in the reverse order. Before the experiment starts, we offer a 10-minute hands-on session, so participants can get used to the device. For both 'Basic' and 'Enhanced' simulators, participants had two driving scenarios: normal free-driving mode, and driving by following another vehicle in the traffic. During the experiment, after each driving task, participants were asked to fill in a questionnaire describing their driving experience in terms of realism and immersion. 
Average rating of importance on different factors

Vehicle lights Indicator sound Traffic dynamics Vehicle color

$\begin{array}{lll}4.08 & 3.75 & 3.25\end{array}$

Table 1: The influence of different factors on the realism of the simulator. (Ratings from 1: least influential to 5: most influential)

We compared the questionnaire results of the two simulators. As shown in Fig. 3, participants in the free-driving mode reported feeling much more engaged in the enhanced simulation. On the other hand, when following another vehi-

Gender of participants

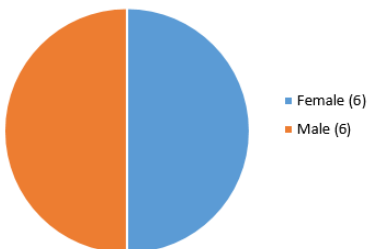

Figure 4: Gender distribution of the participants

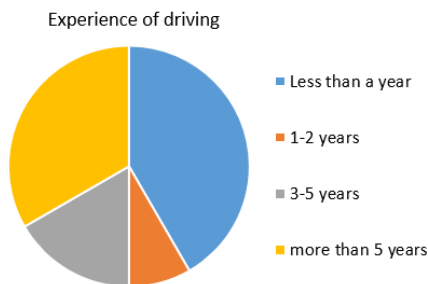

Figure 5: Driving experience of the participants cle, there was less strong preference from the participants.

This may be explained by drivers concentrating on a narrow view due to the immediate task of following, and so being less affected by changes in the surrounding traffic. This is also reflected in their infraction logs: in both simulations, participants tend to commit more traffic infractions when following the other vehicle. This may suggest that the workload of this additional task may be beyond the driver's ability to have a broad perception and to drive safely.

When asked if they feel immersed in the driving environment, participants' responses showed that the enhancements improved their immersion experience as shown in Fig. 6. Moreover, among the factors that differed between the two simulations (see Table 1), the colours of the surrounding vehicles - as expected - were found to be the least influential on the drivers' assessment of the simulator's realism. The other three factors have proved to be of similar importance.

At the end of experiment, we collected participants' feedback about the experiment. It was obvious that the resistance/flexibility of the controller is different from a rea vehicle. Also the missing sensation of movement on the body was reported to affect the participants' immersion in the driving scene. Improving the simulation equipment for
Histogram of ratings on the level of environment realism

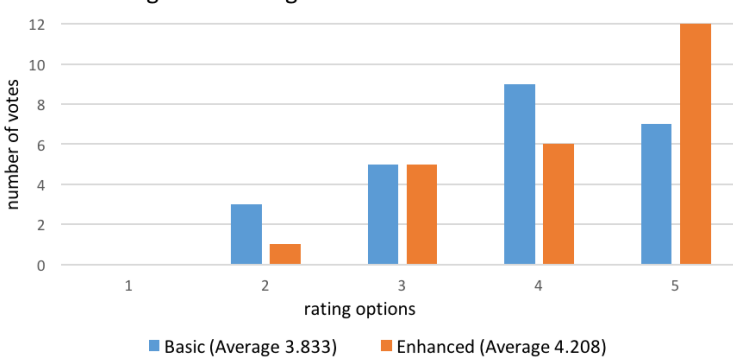

Figure 6: The enhanced simulator increases the level of environment realism reported by the drivers $(p=0.058)$

greater physical engagement of the driver is still a challenging factor in our research.

\section{Conclusion}

This paper presents our developments to enhance an Open Source simulation platform which is specialized for driving experience studies. Our preliminary experiments show that when the scenario includes more human factors, people tend to behave as if they are driving in a real traffic flow rather than a video game. Additionally, the developments allow drivers to observe each other's behaviour. From their reaction and comments on other vehicles, we hope to understand how human drivers judge the traffic situation, and transfer this knowledge to advanced driving assistance systems. For future work, we will extend our evaluation to collect data from more participants, and assess the relative contributions of each factor. Applying our proposed traffic enhancements to more sophisticated and visually-realistic driving simulators is also envisaged, if the high cost of such simulators can be overcome.

\section{Acknowledgement}

This work is funded and supported by Jaguar Land Rover. 


\section{REFERENCES}

1. GENIVI Alliance. GENIVI driving simulator. https : //github.com/GENIVI/genivi-vehicle-simulator.

2. Jusuf Çapalar and Cristina Olaverri-Monreal. 2017. Hypovigilance in limited self-driving automation: Peripheral visual stimulus for a balanced level of automation and cognitive workload. In 2017 IEEE 20th International Conference on Intelligent Transportation Systems (ITSC). 27-31.

3. Alexey Dosovitskiy, German Ros, Felipe Codevilla, Antonio Lopez, and Vladlen Koltun. 2017. CARLA: An Open Urban Driving Simulator. In Proceedings of the 1st Annual Conference on Robot Learning. 1-16.

4. Despina Michael, Marios Kleanthous, Marinos Savva Smaragda Christodoulou, Maria Pampaka, and Andreas Gregoriades. 2014. Impact of immersion and realism in driving simulator studies. International Journal of Interdisciplinary Telecommunications and Networking (IJITN) 6, 1 (2014), 10-25.
5. Michael G Wade and Curtis Hammond. 1998. Simulation Validation: Evaluating Driver Performance in Simulation and the Real World. (1998).

6. Erroll Wood. 2017. Warp-and-Blend-Quadros. https: //github.com/errollw/Warp-and-Blend-Quadros. (2017).

7. Jing Zhao, Meiping Yun, H Michael Zhang, and Xiaoguang Yang. 2015. Driving simulator evaluation of drivers' response to intersections with dynamic use of exit-lanes for left-turn. Accident Analysis \& Prevention 81 (2015), 107-119.

8. Ilka Zöller, Bettina Abendroth, and Ralph Bruder. 2017. Driver behaviour validity in driving simulators - Analysis of the moment of initiation of braking at urban intersections. Transportation Research Part F: Traffic Psychology and Behaviour (2017). 